\title{
Control System Design and Upgrade Considerations for the Duke Free Electron Laser Laboratory
}

\author{
S. Hartman*, V. N. Litvinenko, G. Swift, I. Pinayev, Duke FEL Lab, Durham, NC 27708, USA \\ Y. Wu, ALS-LBNL, Berkeley, CA 94720, USA
}

\begin{abstract}
The Duke Free Electron Laser Laboratory currently operates two FELs: the OK-4/Duke Storage Ring FEL, and the Mark III IR FEL. Both of these systems were among the early adopters of the Experimental Physics and Industrial Control System (EPICS). The Mark III EPICS control system was completed and operational in September, 1993. The Duke Storage Ring was commissioned in November of 1994 using EPICS. These control systems have proven to be very robust with high reliability over the intervening years. An overview of these current control systems and an upgrade strategy will be presented.
\end{abstract}

\section{INTRODUCTION}

\subsection{Mark III}

The Mark III FEL is a tunable, mid-infrared radiation source associated with a $40 \mathrm{MeV}$ linac. A previous control system for the Mark III was replaced with an EPICS based system in September, 1993 [1]. The control system utilized EPICS release 3.9 with all I/O over a CAMAC bus which had been in use with the previous control system. The control system consisted of one VME Input/Output Controller (IOC) connected to a single CAMAC crate.

\subsection{Duke Storage Ring and Linac}

The Duke Storage Ring is a $1 \mathrm{GeV}$ electron storage ring designed for driving UV-VUV free electron lasers. The storage ring was commissioned in November of 1994 using EPICS. The storage ring utilized EPICS release 3.11 with a mixed architecture of CAMAC, Allen-Bradley, VME and GPIB hardware modules distributed over two VME IOCs with 1,423 EPICS records [2]. Its associated $270 \mathrm{MeV}$ linac injector also used EPICS release 3.11 with all I/O over a CAMAC bus.

\section{CURRENT STATUS}

\subsection{Network Topology}

The Duke storage ring and linac control system currently consists of four VME IOCs plus three Sun workstations providing operator interface and high level controls. The Mark III system is independent of the storage ring system and consists of one VME IOC plus one Sun workstation. Additional Sun and Linux workstations and an additional

\footnotetext{
*hartman@fel.duke.edu
}

VME IOC are used for development, testing and supplemental interface. All network traffic is over category 5 twisted pair ethernet wires using the EPICS Channel Access Protocol over TCP/IP. A switched network backplane provides 10/100 Mbps switched ports for each network node, though most control system nodes currently operate at $10 \mathrm{Mbps}$. Numerous additional network ports are available in the ring and laboratory areas to allow a portable Linux workstation to be installed temporarily for diagnostics, testing or monitoring of the systems.

\subsection{Mark III}

The EPICS based control system for the Mark III FEL has changed little over the intervening years. The system currently consists of one VME IOC with 436 EPICS records. All I/O is over a CAMAC bus. Additional information on recent and future Mark III upgrades can be found in [3]. Control system upgrades, including a timing system and new I/O modules, will take place along with hardware upgrades. The system will utilize EPICS release 3.13.4 after these upgrades.

\subsection{Storage Ring and Linac}

The storage ring and linac systems are currently using both EPICS release 3.11 and release 3.13.4. We plan to standardize on release 3.13.4 which is the current stable release of EPICS.

The system currently consists of four VME IOCs with Motorola MC680x0 processors running the VxWorks RealTime Operating System. There are presently 2,329 EPICS records. The primary hardware interface in use is CAMAC with six CAMAC crates connected via a serial branch driver to three of the VME crates. We have a significant amount of CAMAC hardware on hand with numerous 16-bit DACs, 14-bit SAMs and 20-bit ADCs. AllenBradley I/O modules are used for slower subsystems such as cooling-water temperature monitoring, vacuum, RF and mechanical screens. These modules are divided among four Allen-Bradley crates connected to one VME IOC by means of an AB-6008SV module. Beam Position monitors are implemented with Bergoz modules connected to VMIVME 3122 ADCs with VMIVME 3419 signal conditioning boards to reduce AC noise [4]. Additionally, a GPIB interface to a HP3458A digital multimeter provides precision readbacks for quadrupole magnets in the storage ring and the linac-to-ring transfer area. 


\subsection{Recent Upgrades}

The operator workstations are Sun SPARC workstations running Solaris 7. They are located in a central control room and accessible over the network from other workstations within the DFELL building. The operator interface for EPICS has recently been reworked using EPICS release 3.13 client programs. The primary graphical user interface is medm version 2.3.6, developed at Argonne National Laboratory. This interface has proven to be more flexible and more intuitive then the original $e d d / d m$ interface. The ability to call shell commands from a medm screen has simplified operator training as complex tasks can be accomplished with a single button click. The EPICS clients StripTool (from Jefferson National Laboratory) and Display Page (from Argonne National Laboratory) have both proven extremely useful in debugging hardware related errors and isolating power supplies not performing to specifications.

We have recently implemented a feedback routine for regulating quadrupole magnets. The storage ring has sixtyfour quadrupole magnets of two types, each fed by an individually controlled power supply. The twenty-one quadrupoles in each of the two arcs are combined function quadrupoles, with independently controllable quadrupole and sextupole moments. Readbacks for both the main current and the shunt regulated current are made using a precision digital multimeter connected to the EPICS system with a GPIB interface. The feedback routine was incorporated into the existing readback routine running on the IOC and provides regulated adjustments to the power supply control signal as individual readbacks are made. By providing a slow feedback to the quadrupole power supplies, we have been able to eliminate a long term drift apparent in some of the power supplies. Moreover, the feedback routine can maintain long term stability of the current to approximately $\pm 0.1 \mathrm{~mA}$ relative to the setpoint. Although the readbacks are too slow for the feedback routine to be able to address ripples, it does offer a significant improvement over the measured long term stability of these power supplies which have shown instabilities on the order of several $\mathrm{mA}$. The use of this feedback routine has greatly increased magnetic lattice reproducibility from run to run, and improved beam lifetime.

A significant amount of our control system's high level control was originally executed using an extended Tcl-Tk program with EPICS channel Access called epicswish [2]. This provided great flexibility in the control system design and development. However, it does have weaknesses in terms of robustness, speed and consistency. We have begun the process of migrating the functionality of the TclTk scripts to the IOC level, either by implementing the same function with EPICS database records or as a compiled subroutine to run on the IOC. We are also evaluating the use of Matlab for some high-level controls which are currently implemented using Tcl-Tk.

\section{FUTURE PLANS}

\subsection{Storage Ring}

We are currently in preparation for an upgrade of the quadrupole power supplies. The present 64 storage ring quadrupole power supplies (powering individual magnets) will be replaced by twenty-two power supplies of two types, powering groups of magnet coils in series. We plan to interface the RS-232 serial control of these power supplies with our control system using SBS IndustryPack Octal 232 modules attached to an SBS VIPC616 carrier board residing in a VME IOC controlled by an existing Motorola MVME167 board. Testing has begun using the EPICS ipac module [5] along with the the mpfSerial Serial Support Facility [6].

We have also developed EPICS hardware support for the VMIVME 3113A scanning 12-bit analog to digital converter module. We plan to use this module for readback for quadrupole trim power supplies. These supplies will be controlled using VMIVME 4100 modules for which EPICS support already exits. This will decrease our reliance on outdated CAMAC DACs and SAMs. However, we will continue to use some CAMAC hardware until we are able to acquire replacement VME based modules with resolution of 16-bits (see Figure 1).

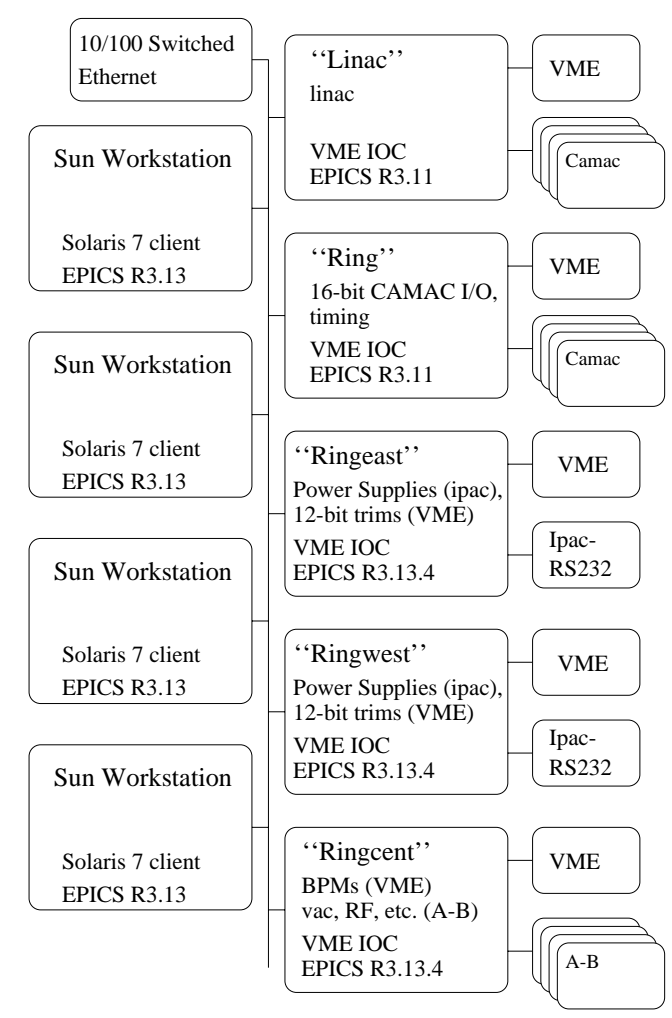

Figure 1: Proposed hardware layout for the Duke Storage Ring control system.

We will take the opportunity of this upgrade period to move more of our IOCs to EPICS release 3.13.4. We will also continue to migrate away from our current high level 
control interface base on Tcl-Tk. New ramping routines will be developed.

\subsection{Vacuum}

The vacuum control subsystem can operate as a standalone system. Readbacks for critical areas are made available to the operators through EPICS using Allen-Bradley based 12-bit analog input modules connected to vacuum gauges and vacuum pumps. The vacuum engineering group makes use of DirectSoft PLCs to monitor a greater number of vacuum readings. This data acquisition and logging is currently handled independently with a Windows PC. We plan to develop a means to integrate the PLCs with EPICS to allow the exchange of data between to two systems.

\section{CONCLUSION}

The Duke FEL Laboratory's control systems have been stable, reliable and easy to operate. We have been able to utilize pre-existing hardware (such as CAMAC) to minimize costs, and we have been able to capitalize on the software development of other members of the EPICS Collaborative. We will continue to develop and expand our existing control system to increase functionality, ease of use, and reliability of the facility.

\section{REFERENCES}

[1] C. Dickey, et al., "EPICS at Duke University," In Dallas 1995, PAC, vol. 4, 2217.

[2] Y. Wu, B. Burnham, and V. N. Litvinenko, "The Duke Storage Ring Control System,” In Dallas 1995, PAC, vol. 4, 2214.

[3] I. Pinayev, et al., "Status of Mark III FEL," These Proceedings.

[4] P. Wang, el al., "Beam position monitors for Duke FEL storage ring," Contributed to IEEE Particle Accelerator Conference (PAC 99), New York, 29 Mar - 2 Apr 1999.

[5] "EPICS ipac Module,"

http://www.aps.anl.gov/asd/people/anj/ipac/.

[6] "EPICS: Serial Support for the Message Passing Facility," http://www.aps.anl.gov/asd/people/mrk/epics/modules/ serial/mpfSerial/. 